\title{
The mass-loss before the end: two luminous blue variables with a collimated stellar wind
} C. Agliozzo $^{1}$, C. Trigilio

${ }^{1}$ Departamento Ciencias Fisicas, Universidad Andres Bello, Av. Republica 252, Santiago, Chile email: c.agliozzo@gmail.com

${ }^{2}$ INAF-Osservatorio Astrofisico di Catania, Via S. Sofia 78, I-95123, Catania, Italy

${ }^{3}$ European Southern Observatory, Alonso de Córdova 3107, Vitacura, Santiago, Chile

${ }^{4}$ National Optical Astronomy Observatory, 950 N Cherry Ave, Tucson, AZ 85719, USA

${ }^{5}$ Núcleo Astronomía Facultad Ingeniería, Universidad Diego Portales, Av. Ejército 441, Santiago, Chile

\begin{abstract}
We gathered a multiwavelength dataset of two well-known LBVs. We found a complex mass-loss, with evidence of variability, such as has been seen previously. In addition, our data reveal signatures of collimated stellar winds. We propose a new scenario for these two stars where the nebula shaping is influenced by the presence of a companion star and/or fast rotation.
\end{abstract}

Keywords. stars: individual (RMC127, HR Car), stars: mass loss, stars: winds, outflows

\section{Introduction}

Recently the classical view of luminous blue variables (LBVs) as a phase of the post-main sequence (MS) evolution of a single massive star has been challenged by Smith \& Tombleson (2015), who proposed that LBVs are mass-gainers in binary systems with Wolf Rayet (WR) stars, which are the mass-donors. Humphreys et al. (2016) defended the accepted description of LBVs as evolved massive stars that have to quickly lose their $\mathrm{H}$ envelope through severe mass-loss, before to evolve as WRs. There is a growing evidence that H-poor Core Collapse-Supernovae ( $\mathrm{SNe}$ ) progenitors consist of both binary and single stars. The mechanism that induces some LBVs to explode as Type IIn $\mathrm{SNe}$ is not known. The debate is very active and the mass-loss mechanism that triggers the LBV Giant Eruptions (Humphreys \& Davidson 1994) is still not understood. Our understanding is made difficult by the rarity of this phenomenon in our Galaxy. On the other hand, we know that at least $70 \%$ of massive stars have to exchange masses with a partner at least once in their life (Sana et al. 2012). Is the asymmetry of several LBV nebulae due to intrinsic asymmetry of the stellar mass-loss or due to the influence of a companion star? What can we learn from resolved imaging by the latest instrumentation? In this talk I will discuss two interesting cases: the LBVs HR Carinae (HR Car) and RMC127.

\section{The Galactic LBV HR Car}

The study I am going to discuss has been published in Buemi et al. 2017. The nebula associated with HR Car has a bipolar filamentary structure seen in $\mathrm{H} \alpha$ by Nota et al. 1997. These authors suggested a similarity with the Homunculus nebula of $\eta$ Car. White 2000 observed an asymmetric nebula emitting in the radio, co-spatial with the optical emission. During the quiescence, HR Car is a B2 supergiant and is not hot enough to ionize the circumstellar nebula, therefore White 2000 suggested that HR Car may have a 

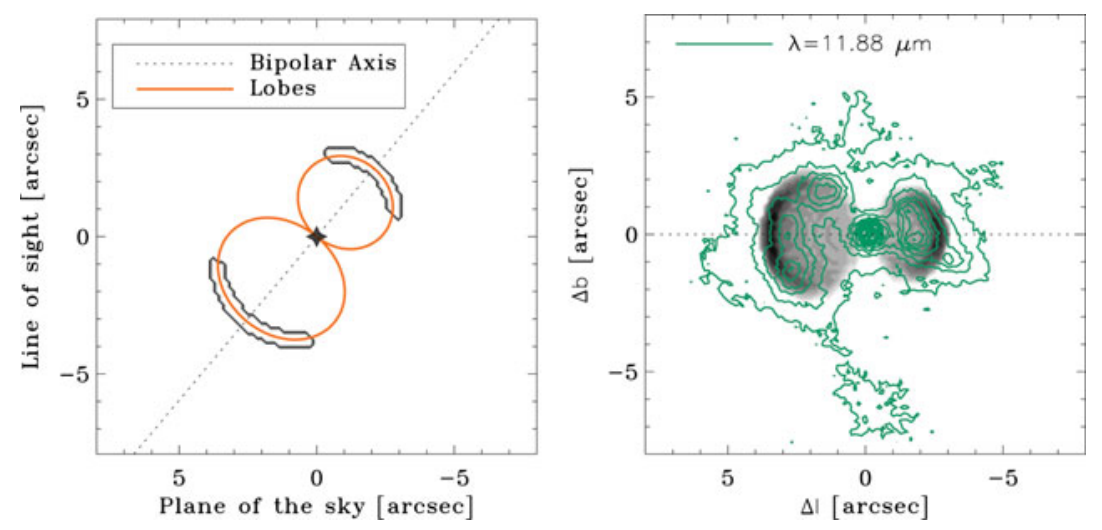

Figure 1. Figure readapted from Buemi et al. 2017. Left panel: schematic representation of the bipolar model for HR Car's nebula. Right panel: simulated emission from the two polar lobes (grey) and VISIR mid-IR emission (green contours) as seen at $11.88 \mu \mathrm{m}$.

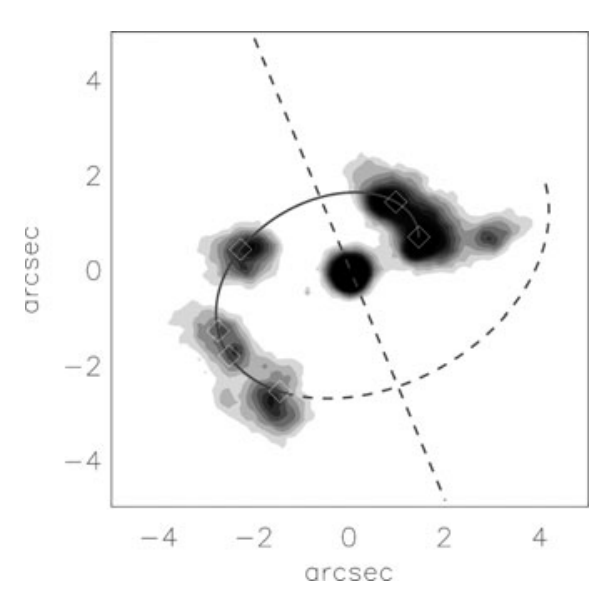

Figure 2. VISIR mid-IR image of the inner dust envelope and spiral model (line). Figure readapted from Buemi et al. 2017.

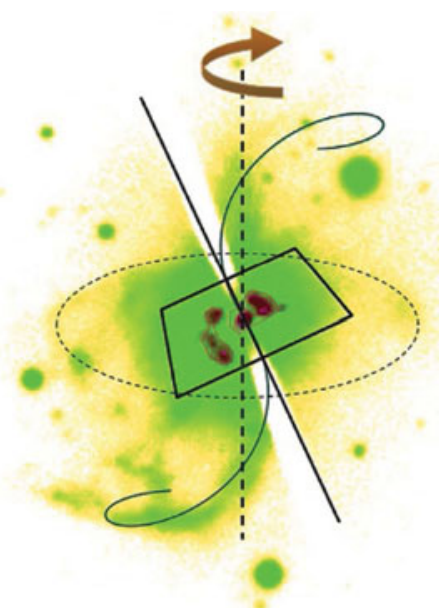

Figure 3. $\mathrm{H} \alpha$ image (green/yellow), VISIR mid-IR image (red), jet-precession model (lines). Figure readapted from Buemi et al. 2017.

companion star responsible for the ionization of HR Car's nebula. Recently, Boffin et al. 2016 obtained a direct detection of at least one companion star for HR Car, very likely a red supergiant (RSG). RSGs do not have sufficiently ionizing photon flux. Therefore, the ionization of the circumstellar material around this system is still unexplained.

In Buemi et al. 2017 we presented a new study of the circumstellar environment of HR Car, based on a multiwavelength dataset. This comprises radio ATCA observations at two different epochs, far-IR Herschel/PACS images and mid-IR VLT/VISIR images. The Herschel images indicate that HR Car's optical and radio nebula is surrounded by an extended and asymmetric outer nebula of optically thin dust.

From the radio data we found that the mass-loss rate increased by a factor of 2 between 1994 and 2014. During the observations at both epochs the star was in the quiescent phase. This variation does not seem correlated with the star S Doradus cycle, so we proposed that it could have been induced by the periastron passage of the companion star, constrained by Boffin et al. 2016 between 2013.2 and 2015.5. 
Our VISIR images show IR emission from an inner dust envelope with a two arc shape (see the green contours in Fig. 1, right panel, and the grey image in Fig. 2). These structures can be fitted with an expanding bipolar-lobe model (Fig. 1), as shown in Buemi et al. 2017, consistently with the model by Nota et al. 1997. In Buemi et al. 2017, we also considered an alternative scenario, where these two arcs close to the star form an Archimedean spiral (Fig. 2). In this case the spiral nebula would be generated by equatorial mass-loss events. The direction of this mass-loss depends on the position of the companion star at the moment of the ejection. In this scenario the ionized gas, that seems confined within this arc-shaped material, is channeled along an axis perpendicular to the plane of the spiral (Fig. 3). Since HR Car is in a binary or even multiple system, the star may suffer a torque that causes an axial precession and then a helical outflow. The stellar wind would be collimated along the rotational axis of HR Car, and gives origin to the $\mathrm{H} \alpha$ and free-free emission. The ionization of the ejecta has to occur thanks to a third companion, a spectral type BO V star, as suggested by White 2000.

\section{The Magellanic LBV RMC127}

RMC127 is another classical LBV in the Large Magellanic Cloud. First optical observations of RMC127's nebula revealed a diamond-shaped nebula (Clampin et al. 1993). Schulte-Ladbeck et al. 1993, and later Davies et al. 2005, found a high-degree of polarization associated with the central star, suggesting an aspherical mass-loss, possibly a disk in the equatorial plane of the star. Later the HST provided the highest resolution image of the $\mathrm{H} \alpha$ nebula (Weis 2003). Weis 2003 found that the nebula deviates from a spherical symmetry, with a bipolarity in the North-South direction, forming two Caps, perpendicular to two Rims in the East-West direction. This configuration resembles the nebular features reproduced by Nota et al. 1995 by simulating a fast outflow in a preexisting dense medium.

We gathered a unique dataset at long wavelengths, consisting of multi-frequency and multi-resolution radio maps acquired with ATCA, and of a sub-millimeter map obtained with ALMA. We also observed RMC127 with VISIR at VLT and with the high-dispersion spectrometer MIKE on the Clay Telescope at Las Campanas Observatory.

In the optical spectrum, acquired in January 2013, we found a broad and shallow photospheric absorption line Si IV $\lambda 4088$, as shown in Fig. 4, which suggests that RMC127 is a fast rotator. From $\mathrm{P}$ Cyg profiles of He I lines with the absorption component saturated we could also determine the terminal velocity of the wind $\left(148 \pm 14 \mathrm{~km} \mathrm{~s}^{-1}\right)$, an important parameter to estimate the mass-loss rate.

Very likely the IR emission detected from the space telescopes arises from extended optically thin dust, rather than a compact shell close to the star. This would explain why we did not obtain any detection with VISIR.

We detected RMC127 in the radio and in the submillimeter. The interferometric maps offer an inside look at the core of the nebula, where the HST image is limited by artifacts due to the bright star. The nebula was detected with ATCA at $1.3 \mathrm{~cm}(17 \mathrm{GHz}), 3.3$ $\mathrm{cm}(9 \mathrm{GHz})$ and $6 \mathrm{~cm}(5 \mathrm{GHz})$, as shown in Fig. 5. In the maps we recognize the two Northern and Southern Caps and the Eastern and Western Rims. In addition, there are diagonal Arms crossing the nebula center. The brightest emission has a "Z" shape and is not reproduced in the simulation by Nota et al. 1995 .

At $9 \mathrm{GHz}$ the central object emission emerges from the nebula and becomes brighter at higher frequencies. At $23 \mathrm{GHz}$ and at the ALMA frequency $(349 \mathrm{GHz})$ this object is the only detected component of emission and it appears as a point source. By fitting the central object flux density distribution from 9 to $349 \mathrm{GHz}$, we found that the spectral 


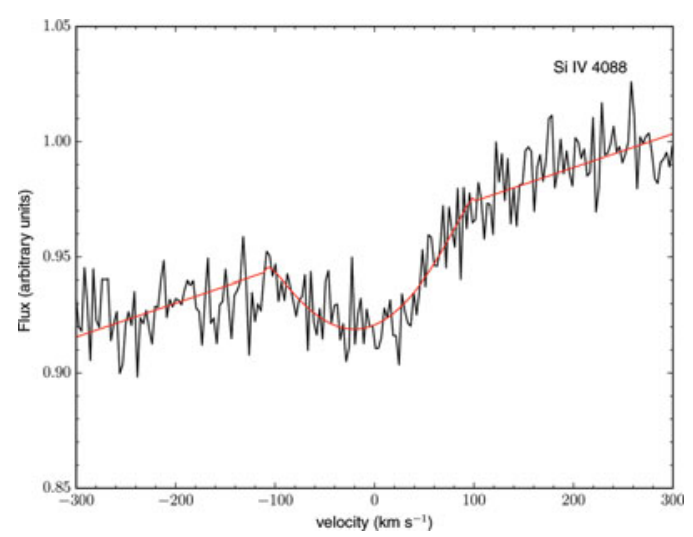

Figure 4. Black line: observed Si IV absorption line in our MIKE spectrum of RMC127 acquired in 2013. Red line: best-fit obtained with a rotational broadening profile. The fit gives a projected rotational velocity of $\sim 105 \mathrm{~km} \mathrm{~s}^{-1}$ and was obtained with a routine in the package PyAstronomy.

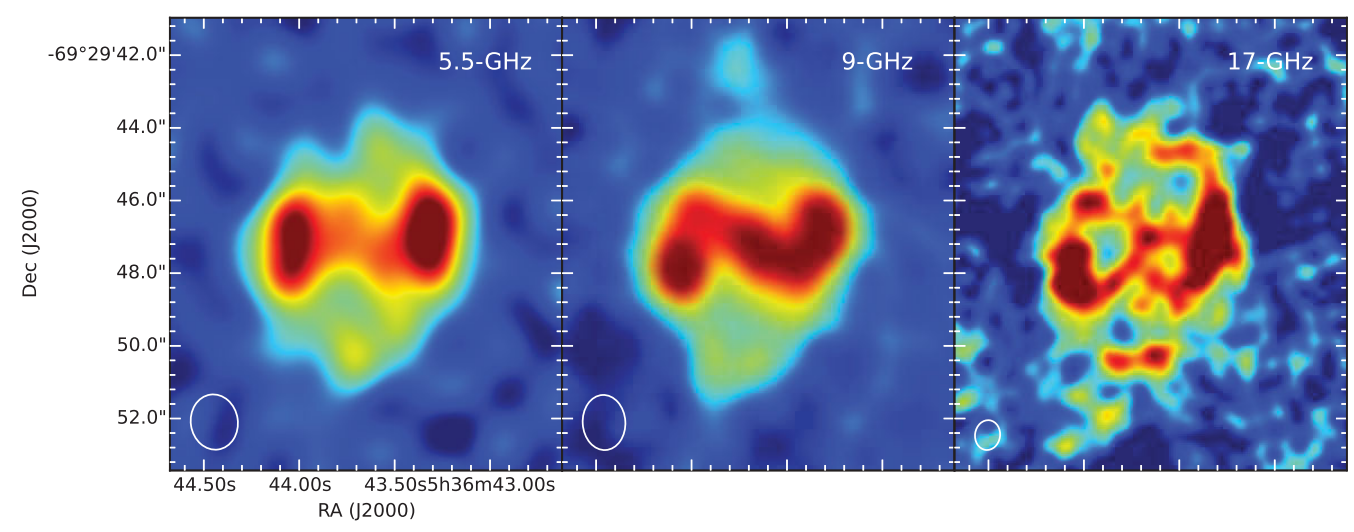

Figure 5. ATCA radio maps at different frequencies and resolutions. The synthesized beam (resolution) in each map is indicated with a white ellipse. $\mathrm{N}$ is up and $\mathrm{E}$ is left.

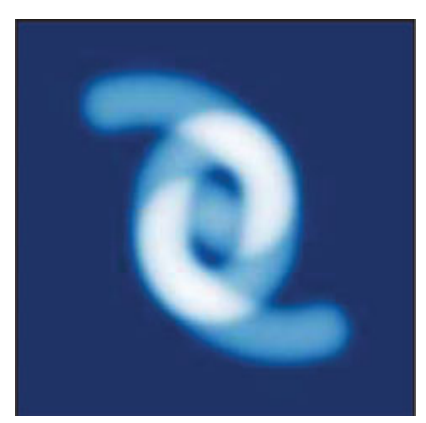

Figure 6. A frame extracted from the simulation of a flight around a 3-D double helix nebula. The simulation was performed by assuming that the axial precession of the star has completed one period. An animated version can be found at http://vimeo.com/151528747.

index $\alpha\left(S_{\nu} \sim \nu^{\alpha}\right)$ is positive $(0.78 \pm 0.05)$, suggesting thermal emission from an ionized stellar wind. However, the high value indicates an excess of emission, departing from the classical case of an ionized spherical wind $(\alpha=0.6)$. We examined different mechanisms that could give origin to such high spectral index. The asymmetries found in RMC127's 
stellar wind and optical nebula by the previously mentioned authors make all the models based on spherical symmetry unsuitable for RMC127. Therefore, we modeled the central object emission with the collimated stellar wind model formulated by Reynolds 1986 . We found that, in this scenario, the mass-loss rate of the outflow would be $\sim 8-9 \times$ $10^{-6} \mathrm{M} \odot \mathrm{yr}^{-1}$, a factor of 3 less than the spherical case.

Collimated stellar winds, jets and bipolar outflows are usually associated with fast rotation and/or dense disks. Such disks can form as a result of mass-transfer in binary systems, magnetic fields or fast stellar rotation. We did not find evidence of a dense disk in our multiwavelength data, but we know that RMC127 is indeed a fast rotator.

Finally, we attempt to explain the Z-shape morphology of the outer nebula with a new geometry. We explored the possibility of a highly inclinated bipolar nebula, as suggested first by Schulte-Ladbeck et al. 1993. Fig. 6 shows a frame extracted from the simulation of a flight around a 3-D double conical helix nebula that we created by using our newly public code RHOCUBE (that allows modelling 3D density distributions and fitting the observed maps; Agliozzo et al. 2017, Nikutta \& Agliozzo 2016). We found remarkable similarities with the radio maps (Fig. 5). This geometry would imply that the nebula was formed by the precession of a bipolar-outflow. The precession needs a torque from a companion star to occur, similarly to HR Car. However, the binarity nature of RMC127 has not been yet demonstrated.

\section{Final comments and outlook}

The circumstellar material around HR Car and its mass-loss seem strongly influenced by the presence of at least one companion star. To explain the nebular morphology, we suggest that RMC127 may be an analogous case to HR Car. The mechanism discussed in these studies seems to us an interesting case to further investigate, to understand the role of binarity in the post-MS evolution of massive stars and the physical phenomenon that triggers the Giant Eruptions in LBVs and in SN impostors. The next step will be the verification of the jet precession scenario with integral field unit observations.

\section{References}

Agliozzo, C., Nikutta, R., Pignata, G., et al. 2017, MNRAS, 466, 213

Boffin, H. M. J., Rivinius, T., Mérand, A., et al. 2016, A\&A, 593, A90

Buemi, C. S., Trigilio, C., Leto, P., et al. 2017, MNRAS, 465, 4147

Clampin, M., Nota, A., Golimowski, D. A., Leitherer, C., \& Durrance, S. T. 1993, ApJ (Letters), 410, L35

Davies, B., Oudmaijer, R. D., \& Vink, J. S. 2005, A\&A A, 439, 1107

Humphreys, R. M. \& Davidson, K. 1994, PASP, 106, 1025

Humphreys, R. M., Weis, K., Davidson, K., \& Gordon, M. S. 2016, ApJ, 825, 64

Nikutta, R. \& Agliozzo, C. 2016, Astrophysics Source Code Library, ascl:1611.009

Nota, A., Livio, M., Clampin, M., \& Schulte-Ladbeck, R. 1995, ApJ, 448, 788

Nota, A., Smith, L., Pasquali, A., Clampin, M., \& Stroud, M. 1997, ApJ, 486, 338

Reynolds, S. P. 1986, ApJ, 304, 713

Sana, H., de Mink, S. E., de Koter, A., et al. 2012, Science, 337, 444

Schulte-Ladbeck, R. E., Leitherer, C., Clayton, G. C., et al. 1993, ApJ, 407, 723

Smith, N. \& Tombleson, R. 2015, MNRAS, 447, 598

Weis, K. 2003, A\&\&A, 408, 205

White, S. M. 2000, ApJ, 539, 851 